\title{
CUIDADOS NO TRANSPORTE AEROMÉDICO DE PACIENTES COM ANEURISMA DE AORTA
}

Andréa Mendes DE OLIVEIRA ${ }^{1}$, Michelle TAVERNA ${ }^{2}$, Felipe Trevisan Matos NOVAK ${ }^{3}$, Heloisa Giccele Claudino NOVAK ${ }^{4}$

\section{RESUMO}

Derivada do grego aneuryneim, a atual definição do aneurisma considera a dilatação permanente e localizada numa artéria que ultrapassa $50 \%$ do seu diâmetro normal podendo evoluir para o óbito frequentemente ocasionada por sua rotura. Cerca de $4,5 \%$ dos homens com mais de 60 anos de idade são portadores assintomáticos do aneurisma de aorta (AA) e no mundo chega a representar $2 \%$ de todas as mortes. 0 transporte aéreo desses pacientes é definido basicamente pela necessidade de cuidados adicionais (assistência ou intervenção especializada), não disponíveis no local onde o paciente se encontra, devendo ser ponderados os benefícios potenciais e os riscos inerentes a exposição ao ambiente hipobárico. Este trabalho se trata de uma pesquisa bibliográfica com levantamento de livros, sites e artigos cujo objetivo foi caracterizar a produção científica acerca do transporte aeromédico de pacientes com aneurisma de aorta em bases de dados, especializadas pela Medical Literature Analysis and Retrieval System Online (MEDLINE) via Pubmed. Ainda existem muitos tabus e anseios acerca do transporte aeromédico do paciente com AA no Brasil e embora diante de um quadro grave como este, a literatura nos mostra que é possível realizar o transporte aéreo de forma segura, desde que, o paciente seja estabilizado adequadamente, monitorado com equipamentos de qualidade e a equipe experiente/ treinada que garanta cuidados essenciais para menores variações hemodinâmicas em voo.

Palavras-chave: transporte aeromédico, aneurisma de aorta.

\section{INTRODUÇÃO}

Derivada do grego aneurysm e que significa alargamento, a sua atual definição considera a dilatação permanente e localizada numa artéria que ultrapassa $50 \%$ do seu diâmetro normal. Sabe-se que atualmente, 4,5\% dos homens com mais de 60 anos de idade são portadores do aneurisma de aorta (AA) e em sua grande maioria, sem sintomas (NORDON, 2009). O AA é responsável por $2 \%$ das mortes no mundo todo. A rotura é o seu desfecho mais comum, e o risco de acontecer é diretamente proporcional ao tamanho do aneurisma. A trombose é acontecimento menos comum e ocorre em cerca de 0,5 a $2 \%$ dos casos (VAQUERO-MORILLO, 2006).

\footnotetext{
1. Enfermeira de Voo Helisul, $1^{\circ}$ Ten da Força Aérea Brasileira, Presidente ABRAERO, Coordenadora do Dep. de Neurociência da ABRAERO. Curitiba, Paraná, Brasil. michelletaverna@hotmail.com

2. Médica de voo Helisul, Pós-graduanda em Transporte e Resgate Aeromédico da Faculdade Inspirar, Curitiba, Paraná, Brasil. andrea.oliveira80@hotmail.com

3. Médico de voo Helisul, Membro do Dep. de Neurociência da ABRAERO, Especialista em Transporte e Resgate Aeromédico pela Faculdade Inspirar, Curitiba, Paraná, Brasil. felipenovak@gmail.com

4. Acadêmica de Medicina das Faculdades Pequeno Príncipe, Curitiba, Paraná, Brasil. heloisanovak@hotmail.com
} 


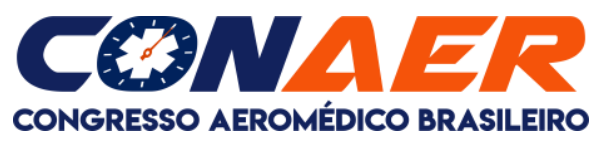

O transporte aéreo de um paciente com aneurisma de aorta é indicado basicamente pela necessidade de cuidados adicionais (assistência ou intervenção especializada), não disponíveis no local onde o paciente se encontra, devendo-se ponderar os benefícios potenciais e os riscos inerentes a exposição ao ambiente hipobárico.

No Brasil esse tipo de transporte é muito frequente, visto o reduzido número de centros especializados. O uso de aeronaves de asa fixa (aviões) ou asa rotativa (helicópteros) visa a intervenção precoce com maior sobrevida, redução do tempo de internação e qualidade de vida nos anos sequenciais. Entretanto, não devemos esquecer que durante os voos os pacientes são expostos a estressores físicos e ambientais, que podem provocar manifestações como taquicardia, bradicardia, arritmias, hipotensão, taquipneia e alteração do nível de consciência (DALAZEN, 2019).

\section{METODOLOGIA}

Este trabalho foi estruturado em uma revisão bibliográfica acerca do transporte aéreo de pacientes acometidos por aneurisma de aorta, a partir da análise crítica e leitura sistemática de livros, sites e artigos científicos selecionados através de busca na base de dados Medical Literature Analysis and Retrieval System Online (MEDLINE) via Pubmed, utilizando as palavras-chave: aortic aneurysm, aerospace medicine, air medical e de bases de dados epidemiológicos como DATASUS. O período de estudo foi de 2002 a 2021, incluindo alguns clássicos literários na temática.

\section{RESULTADOS E DISCUSSÕES}

O aneurisma da aorta apresenta espectro de gravidade considerável. Embora alguns autores sugiram um risco teórico de ruptura relacionado às viagens aéreas ou em voo, essa afirmação permanece questionada pela não comprovação. Na verdade, há poucos dados para fazer recomendações baseadas em evidências. A evacuação médica aérea de um paciente com AA em risco de ruptura iminente ou estado pósruptura recente pode ser realizada, presumindo-se que o cuidado cirúrgico local não esteja disponível e que a transferência esteja levando o paciente a um nível superior de intervenção médica (BARROS, 2014).

Um dos trabalhos de interesse encontrados nesta revisão foi uma retrospectiva de 4 anos dos registros de missão aeromédica interrelacionada aos registros hospitalares. 


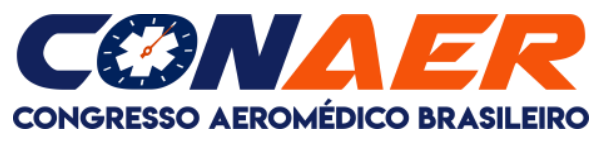

Levou em consideração suspeita de emergência aórtica (não traumática) num total de 34 pacientes dos quais 31 (91\%) chegaram vivos ao hospital. Os diagnósticos na admissão ao hospital: 14 (41\%) foram Aneurisma de Aorta Abdominal Roto, cinco (15\%) Aneurismas de Aorta Abdominal sem ruptura, oito (24\%) dissecções de aorta e quatro $(12 \%)$ sem patologia da aorta. Além disso, 25 pacientes $(74 \%)$ chegaram hemodinamicamente estáveis e dezessete pacientes $(50 \%)$ receberam intervenção cirúrgica de emergência (MITCHELL, 2002). Em outro artigo, de 2015 aplicado no oeste dos Estados Unidos, conduzida por especialistas do Reino Unido, tomou por base pacientes transportados com aneurisma de aorta roto e apontou um aumento na taxa de mortalidade desses pacientes (MELL, SCHNEIDER, e STARNES, 2015).

Em um estudo de DALAZEN (2019) com um total de 67 pacientes transportados analisou a pressão arterial média (PAM), frequência cardíaca (FC) e saturação parcial de oxigênio (SPO2) e em sua conclusão houve pequena variação dos fatores hemodinâmicos analisados, embora expondo os pacientes aos estressores de voo. Para os autores, este achado foi relacionado diretamente a uma boa estabilização da vítima através dos tratamentos empreendidos pelos membros da equipe.

No início da desaceleração para o procedimento de descida da aeronave de asa fixa, a tendência é os fluidos corporais que estão nos membros serem impulsionados para a parte alta do corpo, causando uma sobrecarga súbita no volume circulatório e aumentando o retorno venoso, o que poderia causar uma falência súbita da hemodinâmica circulatória e trazer repercussões indesejadas para os pacientes (BLANCO e TEIXEIRA, 2021).

$\mathrm{Na}$ intenção de minimizar possíveis intercorrências as missões exigem um acurado planejamento de todas as suas etapas operacionais, contando com a habilidade da equipe de voo, o que levará à maiores possibilidades de êxito. O planejamento deve incluir: uma triagem prévia completa, o preparo da aeronave, briefing com a tripulação sobre as limitações e alternativas de correções, a captação do paciente com seu preparo e monitorização em equipamentos adequados para o voo, embarque cauteloso, meticuloso acompanhamento das alterações hemodinâmicas durante todo o voo, desembarque e entrega do paciente com detalhada documentação e relatório de todo percurso (MITCHEL, 2015; BLANCO e TEIXEIRA, 2021). Os autores acrescentam que para minimizar estes efeitos a equipe de voo devem considerar 
cuidados como elevação da cabeceira do paciente para $30^{\circ}$, solicitar aos pilotos que façam uma rampa de descida suave e utilizem o máximo da pista no pouso, evitando a utilização do reverso da aeronave, ou, se for possível, mudar a posição do paciente, colocando a sua cabeça voltada para a cauda da aeronave.

Já nos helicópteros a decolagem e o pouso são feitos na vertical, porém a aceleração favorece o aumento do retorno venoso pelo posicionamento do paciente dentro da aeronave, já que a cabeça estará voltada para a cauda, podendo induzir aumento do sangramento. Entretanto, na desaceleração, esta situação melhora com a diminuição do retorno venoso (BLANCO e TEIXEIRA, 2021).

Com isso, podemos observar que embora diante de um quadro grave como 0 aneurisma de aorta, é possível realizar o transporte aéreo de forma segura, desde que, o paciente seja estabilizado adequadamente, monitorado e a equipe garanta cuidados essenciais para menores variações hemodinâmicas em voo. Diante do exposto é indiscutível a nítida necessidade e importância da capacitação de toda a equipe para exercer a atividade aeromédica. Além das habilidades manuais, técnicas, científicas e intelectuais para o manejo assertivo das emergências e das possíveis alterações ocasionadas pelo ambiente hipobárico.

\section{CONSIDERAÇÕES FINAIS}

Emergências aórticas não são raras em nosso país. Segundo MITCHEL (2002) as políticas e procedimentos específicos baseados na revisão contínua da qualidade devem ser implementados baseadas em dados palpáveis para otimizar o transporte e o cuidado em voo desses pacientes.

Apesar de todas as complicações pré-existentes de um paciente gravemente enfermo, as aeronaves, de uma forma geral, contribuem para a evacuação destes pacientes de locais com precariedade de recursos para ingressarem em um mundo maior de alternativas de tratamento nos grandes centros. Quando se fala resgate/remoção aérea associada ao adequado suporte durante o transporte com equipamentos e sistema de unidade de terapia intensiva móvel ágil estamos aumentando consideravelmente as chances de sobrevida bem como a redução de sequelas permanentes ou temporárias ocasionadas pelo aneurisma de aorta. Por tal motivo, devemos transpor barreiras, com segurança, em busca de todos os meios possíveis para executar estas missões. 


\section{CONAER \\ CONGRESSO AEROMÉDICO BRASILEIRO}

\section{REFERÊNCIAS}

BARROS A., et al. Air travel of patients with abdominal aortic aneurysm: urgent air medical evacuation and nonurgent commercial air repatriation. Air Med Journal, 2014.

BLANCO, DA e TEIXEIRA, WA. Transporte e Resgate em Patologias Cardiológicas. In: SUEOKA,JS. FREIXO, JAA;e TAVERNA M. Transporte e Resgate Aeromédico. Guanabara Koogan. Rio de Janeiro, 2021.

DALAZEN AB; UEZ, GC e BARCALA, RS. Repercussões hemodinâmicas nos pacientes transportados por helicótero no Oeste Caterinense. Congresso Aeromédico Brasileiro 2019.

MELL, MW, SCHNEIDER, PA e STARNES, BW. Variability in transfer criteria for patients with ruptured abdominal aortic aneurysm in the western United States. Presented at the Twenty-ninth Annual Meeting of the Western Vascular Society, Loews Coronado, 2014. Publicado em Air Med Journal, 2015.

MITCHELL, AD, TALLON, MJ. Air medical transport of suspected aortic emergencies. Air Med Journal, 2002.

NORDON IM; et al. Review of current theories for abdominal aortic aneurysm pathogenesis. Vascular 2009.

VAQUERO-MORILLO F. Aneurismas aortoilíacos. In:Tratado de las Enfermedades Vasculares. Barcelona:Viguera; 2006. p. 843-78 